\title{
Estudiantes de medicina en riesgo: prevalencia e incidencia de conversión de PPD
}

\author{
Kocfa Chung-Delgado, Sonia Guillén-Bravo, Laura Navarro-Huamán, Rafael Quiroz-Portella, \\ Alejandro Revilla-Montag, Andrea Ruíz-Alejos, Mariana Zapata-Pachas y Antonio Bernabé-Ortiz
}

\section{Medical students at risk: prevalence and incidence of tuberculin skin test conversion}

Introduction: One third of the world population is affected by latent tuberculosis, with 9.4 million new cases; medical students have 2 to 50 times the probability of acquiring the infection. Objectives: Establish the baseline prevalence of positive tuberculin skin test (TST) at the beginning of medical studies and determine the incidence and variables associated with TST conversion in medical students. Materials and Methods: Secondary analysis of a historical cohort (2007-2010) of medical students in a private Peruvian university. The TST conversion was evaluated. A binomial regression analysis was applied for each associated variable. Results: 707 medical students were included, of whom 219 (31\%) were male. The basal prevalence of reactive TST was 14.4\%. Significant associations were found with the year of university entry of 2007-08 $(p=0.007)$ and a history of tuberculosis $(\mathrm{p}=0.02)$. With a total of 822 person-years, the incidence of conversion was 2.92 cases per 100 person-years (CI95\%: 1.96-4.36). The TST conversion was associated with the year of university entry (RR $=2,55$; IC95\%: $1,06-6,30)$ and a body mass index $\geq 25 \mathrm{~kg} / \mathrm{m}^{2}$ ( $\mathrm{RR}=0,16$; IC95\%: 0,01-0,97). No association was detected with gender, tobacco or alcohol use. Conclusions: There is evidence of a high basal prevalence of latent tuberculosis infection in medical students. The incidence rate is within expected values and high in comparison with the general population. People with $\mathrm{BMI} \geq 25 \mathrm{~kg} / \mathrm{m}^{2}$ have protection against a latent infection. In medical students, more attention should be paid to biosecurity.

Key words: Tuberculosis, Pulmonary / Peru Epidemiology / Students, Medical / Tuberculin Test.

Palabras clave: Tuberculosis pulmonar, Perú/epidemiología, estudiantes de medicina, prueba de tuberculina.

\section{Introducción}

A pesar del esfuerzo realizado por detener la tuberculosis (TBC) en el mundo, esta enfermedad sigue siendo un problema de salud pública. Según datos de la Organización Mundial de la Salud (OMS), aproximadamente un tercio de la población mundial presenta infección tuberculosa latente ${ }^{1}$. Para el año 2009, se notificaron alrededor de 9,4 millones de casos incidentes de TBC en el mundo y cerca de 1.3 millones de muertes, equivalente a una letalidad de 20 muertes por 100.000 habitantes: la segunda causa más frecuente de mortalidad por infección ${ }^{1,2}$.

Según la OMS y el Ministerio de Salud del Perú, alrededor de 35 mil casos de TBC son diagnosticados cada año, una de las cifras más elevadas en el concierto mundial $^{1}$. En el año 2009, las tasas de morbilidad e incidencia fueron 118 y 103 por 100,000 habitantes, respectivamente, con altas tasas de formas multidrogoresistentes (MDR) y la aparición cada vez más frecuente de formas extremadamente-drogo-resistentes (XDR), lo que podría explicar la tasa de mortalidad de 3,2 por cada 100.000 habitantes $^{3}$.
Cada persona que desarrolla la enfermedad pulmonar por el bacilo tuberculoso puede infectar de 10 a 15 personas al año ${ }^{4,5}$. Así, aproximadamente cinco personas se infectan del bacilo tuberculoso cada hora en el Perú3. De éstos, 5 a 10\% llegará a desarrollar la enfermedad completa con cuadro clínico de $\mathrm{TBC}^{3,4}$. Alrededor de 5\% de los infectados desarrollan la enfermedad activa durante el primer año; mientras que el resto la desarrollará en los años posteriores ${ }^{6}$. Más aún, 5,3\% de los nuevos casos adquieren formas resistentes de tuberculosis i $^{3,7,8}$.

La alta posibilidad de contagio se evidencia principalmente en los trabajadores de salud, que tienen 2 a 50 veces más probabilidad de adquirir la infección en sus centros de trabajo ${ }^{9-12}$. Esta amenaza se extiende hacia los estudiantes de medicina que también se desarrollan en un ambiente de alto riesgo de contagio. La introducción de los estudiantes en el contexto hospitalario les permite el desarrollo de habilidades clínicas y complementar los conceptos recibidos dentro de su formación teórica; sin embargo, ello implica una mayor exposición a diferentes patógenos como Mycobacterium tuberculosis, lo que supone un riesgo de contagio de los mismos. En general, los alumnos toman pocas medidas de bioseguridad para
Universidad Peruana de Ciencias Aplicadas-UPC, Lima, Perú. Escuela de Medicina Sociedad Científica de Estudiantes de Medicina (KCD, SGB, LNH, RQP, ARM, ARA, MZP, ABO). Masters in Public Health and Epidemiology (ABO).

Investigación sin conflicto de intereses.

Investigación auto-financiada.

Recibido: 14 de julio de 2011 Aceptado: 11 de mayo de 2012

Correspondencia a: Kocfa Chung-Delgado kocfachung@gmail.com 
prevenir su contagio, lo que los hace vulnerables a la infección ${ }^{13,14}$.

El objetivo de este trabajo fue evaluar la prevalencia de la infección latente por TBC, medido a través de la prueba de tuberculina (PPD), así como la incidencia de la conversión a la prueba en los diferentes años de estudio en estudiantes de medicina de una universidad privada de Lima, Perú. Asimismo, se evaluó, los posibles factores de riesgo asociados a la prevalencia, así como a la incidencia de conversión de PPD.

\section{Materiales y Métodos}

Diseño y Participantes. Este es un estudio observacional, analítico, de tipo cohorte retrospectiva, que incluyó a los estudiantes matriculados en la Escuela de Medicina de la Universidad Peruana de Ciencias Aplicadas en Lima entre los años 2007 y 2010. Los criterios de inclusión fueron:

- ser estudiante matriculado en la Escuela de Medicina al momento en que se realizó la recolección de los datos $\mathrm{y}$

- haber consentido el uso de su información clínica para el análisis.

Recolección de datos. Este es un análisis de datos secundarios obtenidos de las historias clínicas de los estudiantes de la Escuela de Medicina. Usualmente, cada año, los estudiantes son sometidos a una evaluación médica completa, la cual incluye la realización de la prueba de tuberculina (PPD o prueba de Mantoux) aplicada usando la técnica estándar: una jeringa de tuberculina de $1 \mathrm{~mL}$ con aguja $26 \mathrm{G} \times 1 / 2(0,45 \times 12 \mathrm{~mm})$ inyectándose una dosis de $0,1 \mathrm{ml}$ de forma intradérmica en la cara anterior del antebrazo de la mano no dominante. Se dieron indicaciones a los estudiantes para prevenir un resultado falsamente positivo. Pasada las 48-72 horas, los alumnos regresaron para realizarse la lectura de la reacción cutánea. Dicha medición, expresada en milímetros, fue realizada por personal capacitado usando una regla milimetrada de plástico.

Definición de variables. La variable principal fue la conversión de PPD definido como un resultado positivo $(\geq 10 \mathrm{~mm})$ a la prueba de tuberculina durante el seguimiento siendo previamente negativo, o un incremento $\geq 10 \mathrm{~mm}$ de la medición al inicio del estudio de acuerdo a los registros médicos existentes ${ }^{15,16}$. El seguimiento se realizó usando información de los resultados médicos de rutina realizados en forma anual a los estudiantes de medicina de la universidad. Otras variables que fueron consideradas para el análisis incluyeron sexo ${ }^{17,18}$, año de ingreso a la carrera (en bienios), antecedente de TBC en el pasado [Sí/No], consumo habitual de tabaco [Sí/No] ${ }^{19-21}$, consumo habitual de alcohol $[\mathrm{Sí} / \mathrm{No}]^{22}$, e índice de masa corporal (IMC) $\left[<25 / \geq 25 \mathrm{~kg} / \mathrm{m}^{2}\right]^{19,23,24}$.

Para la estimación de la incidencia se calculó el tiempo hasta la conversión. Para esto, primero se excluyó a todos aquellos que tenían lectura positiva (PPD positivo) al inicio de la carrera. Posteriormente, se estableció que ante la aparición de un resultado positivo de PPD, se debería tener al menos un resultado negativo en el año previo. Para el caso de resultado negativo, como mínimo se requería que el último resultado de lectura fuera negativo, sin necesidad de tener todas las lecturas de los años anteriores.

Análisis estadístico. El ingreso de los datos fue realizado mediante doble digitación usando Microsoft Excel 2007 para Windows. Si se hallaba alguna incongruencia, se contrastaron los hallazgos con los registros originales de los exámenes médicos. Se utilizó STATA versión 11.0 para Windows para el análisis estadístico (Stata Corp, College Station, TX, US).

Inicialmente, se realizó la descripción de las variables de interés de acuerdo a si los participantes tenían PPD positivo o negativo al ingreso a la carrera. Se usó la prueba exacta de Fisher para la comparación de variables categóricas. Luego, se utilizó el análisis de regresión logística para determinar los factores independientemente asociados con un resultado PPD positivo al inicio de la carrera, reportándose odds ratios (OR) e intervalos de confianza al 95\% (IC95\%). Posteriormente, usando la parte de seguimiento de los datos, se calculó la incidencia de conversión de PPD para cada variable de interés usando regresión binomial, reportándose riesgos relativos (RR) así como los IC95\% ajustando por sexo.

Ética. El estudio fue aprobado por la Escuela de Medicina de la Universidad Peruana de Ciencias Aplicadas UPC. Se usó un consentimiento informado escrito para obtener la aprobación de los estudiantes y poder usar sus respectivos registros médicos para el estudio. Todos los nombres e identificadores personales fueron eliminados de la base de datos para mantener la confidencialidad de los estudiantes.

\section{Resultados}

Descripción de los participantes. Un total de 707 estudiantes de medicina (31\% varones) fueron enrolados después de dar consentimiento, con una tasa de rechazo de 1\%. El 35\% de los estudiantes ingresó en el periodo 2007-2008, mientras que el resto ingresó en el periodo 2009-2010.

Prevalencia y factores asociados a PPD positivo al inicio de la carrera. Del total de enrolados, sólo 547 


\begin{tabular}{|c|c|c|c|}
\hline Variables & $\begin{array}{l}\text { PPD negativo } \\
(n=468)\end{array}$ & $\begin{array}{l}\text { PPD positivo } \\
(\mathrm{n}=79)\end{array}$ & $p^{*}$ \\
\hline \multicolumn{4}{|l|}{ Sexo } \\
\hline Mujer & $318(85,7 \%)$ & $53(14,3 \%)$ & \multirow{2}{*}{0,90} \\
\hline Varón & $150(85,2 \%)$ & $26(14,8 \%)$ & \\
\hline \multicolumn{4}{|c|}{ Año de Ingreso a la carrera } \\
\hline $2007-2008$ & $150(79,8 \%)$ & $38(20,2 \%)$ & \multirow{2}{*}{0,007} \\
\hline $2009-2010$ & $318(88,6 \%)$ & $41(11,4 \%)$ & \\
\hline \multicolumn{4}{|c|}{ Antecedente previo de TBC } \\
\hline No & $466(86,0 \%)$ & $76(14,0 \%)$ & \multirow{2}{*}{0,02} \\
\hline Sí & $2(40 \%)$ & $3(60 \%)$ & \\
\hline \multicolumn{4}{|c|}{ Consumo habitual de tabaco } \\
\hline No & $412(85,3 \%)$ & $71(14,70 \%)$ & \multirow{2}{*}{0,83} \\
\hline Sí & $42(87,5 \%)$ & $6(12,5 \%)$ & \\
\hline \multicolumn{4}{|c|}{ Consumo habitual de alcohol } \\
\hline No & $376(87,2 \%)$ & $55(12,8 \%)$ & \multirow{2}{*}{1,0} \\
\hline Sí & $44(88,0 \%)$ & $6(12,0 \%)$ & \\
\hline \multicolumn{4}{|l|}{ Índice de masa corporal 3} \\
\hline Normal & $364(86,1 \%)$ & $59(13,9 \%)$ & \multirow{2}{*}{0,46} \\
\hline Sobrepeso/obesidad & $99(83,2 \%)$ & $20(16,8 \%)$ & \\
\hline
\end{tabular}

\begin{tabular}{|c|c|c|}
\hline Variables & $\begin{array}{l}\text { Modelo bivariado } \\
\text { OR (IC95\%) }\end{array}$ & $\begin{array}{l}\text { Modelo multivariado* } \\
\text { OR (IC95\%) }\end{array}$ \\
\hline \multicolumn{3}{|l|}{ Sexo } \\
\hline Mujer & 1 & \\
\hline Varón & $1,04(0,63-1,73)$ & \\
\hline \multicolumn{3}{|c|}{ Año de ingreso a la carrera } \\
\hline $2007-2008$ & 1 & 1 \\
\hline $2009-2010$ & $0,51(0,31-0,82)$ & $0,47(0,29-0,77)$ \\
\hline \multicolumn{3}{|l|}{ Antecedente de TBC } \\
\hline No & 1 & 1 \\
\hline Sí & $9,20(1,51-55,95)$ & $12,47(2,02-77,03)$ \\
\hline \multicolumn{3}{|c|}{ Consumo habitual de tabaco } \\
\hline No & 1 & \\
\hline Sí & $0,83(0,34-2,02)$ & \\
\hline \multicolumn{3}{|c|}{ Consumo habitual de alcohol } \\
\hline No & 1 & \\
\hline Sí & $0,93(0,38-2,29)$ & \\
\hline \multicolumn{3}{|c|}{ Índice de masa corporal 3} \\
\hline Normal & 1 & \\
\hline Sobrepeso/obesidad & $1,25(0,72-2,17)$ & \\
\hline
\end{tabular}

$(77,4 \%)$ tenían lectura de PPD disponible al inicio de la carrera. De ellos, 79 fueron positivos, con una prevalencia basal de 14,4\% (IC95\%: 11,5-17,4). Los factores asociados a PPD positivo al inicio de la carrera fueron: año de ingreso $(p=0,007)$ y antecedente de TBC $(\mathrm{p}=0,02)$ (Tabla 1$)$.

En el análisis multivariado, se encontró que aquellos que ingresaron en el periodo 2009-2010 tuvieron menor probabilidad de TBC latente $(\mathrm{OR}=0,47$; IC95\%: 0,29$0,77)$ que aquellos que ingresaron en el periodo 20072008; mientras que aquellos con antecedente previo de TBC tuvieron mayor probabilidad de ser PPD positivos $(\mathrm{OR}=12,47$; IC95\%: 2,02-77,0) (Tabla 2).

Incidencia y factores de riesgo para conversión de $P P D$. Un total de 505 estudiantes tuvieron datos para el cálculo de la incidencia, involucrando 822 personasaño de seguimiento. Durante este periodo, 24 personas experimentaron conversión del PPD, con una incidencia de 2,92 (IC95\% 1,96-4,36) por 100 personas-año. La incidencia por cada variable de interés se encuentra en la Tabla 3. Es importante notar que en el análisis bivariado se encontró que haber ingresado a la carrera en el período 2009-2010 fue un factor de riesgo $(\mathrm{RR}=2,55$; IC95\%: 1,06-6,29) comparado con aquellos que ingresaron en el período 2007-2008; mientras que tener un IMC $\geq 25 \mathrm{~kg}$ / $\mathrm{m}^{2}$ en el ingreso de la carrera fue un factor protector
(RR =0,16; IC95\%: 0,01-0,97) comparado con aquellos con IMC $<25 \mathrm{~kg} / \mathrm{m}^{2}$. Sin embargo, sólo el año de ingreso a la carrera mostró ser un factor de riesgo en el modelo multivariado (RR =2,53; IC95\%: 1,11-5,76) (Tabla 3).

Tabla 3. Incidencia y factores asociados a conversión de PPD durante el seguimiento

\begin{tabular}{|c|c|c|c|}
\hline Variables & $\begin{array}{c}\text { Incidencia x } 100 \\
\text { (IC95\%) }\end{array}$ & $\begin{array}{l}\text { Modelo bivariado } \\
\text { RR (IC95\%) }\end{array}$ & $\begin{array}{c}\text { Modelo } \\
\text { multivariado* } \\
\text { RR (IC95\%) }\end{array}$ \\
\hline \multicolumn{4}{|l|}{ Sexo } \\
\hline Mujer & $3,19(2,01-5,07)$ & \multirow{2}{*}{$0,73(0,24-1,92)$} & \\
\hline Varón & $2,33(1,04-5,18)$ & & \\
\hline \multicolumn{4}{|c|}{ Año de ingreso a la carrera } \\
\hline $2007-2008$ & $1,96(1,08-3,53)$ & \multirow{2}{*}{$2,55(1,06-6,29)$} & \multirow{2}{*}{$2,53(1,11-5,76)$} \\
\hline $2009-2010$ & $5,00(2,90-8,61)$ & & \\
\hline \multicolumn{4}{|c|}{ Consumo habitual de tabaco } \\
\hline No & $2,95(1,92-4,52)$ & \multirow{2}{*}{$0,57(0,01-3,57)$} & \multirow{2}{*}{$0,59(0,08-4,29)$} \\
\hline Sí & $1,69(0,24-12,03)$ & & \\
\hline \multicolumn{4}{|c|}{ Consumo habitual de alcohol } \\
\hline No & $3,42(2,25-5,20)$ & & \multirow{2}{*}{ - } \\
\hline Sí & - & & \\
\hline \multicolumn{4}{|l|}{ Índice de masa corporal } \\
\hline Normal & $3,48(2,29-5,29)$ & \multirow{2}{*}{$0,16(0,01-0,97)$} & \multirow{2}{*}{$0,17(0,02-1,18)$} \\
\hline Sobrepeso/obesidad & $0,55(0,08-3,88)$ & & \\
\hline
\end{tabular}

*Resultados mostrados fueron ajustados por sexo. 


\section{Discusión}

En este estudio de cohorte retrospectiva, 3 de cada 100 estudiantes en promedio sufrieron conversión a la prueba de tuberculina anualmente. El ingreso reciente a la Escuela de Medicina -período 2009-2010 comparado con el periodo 2007-2008- y tener diagnóstico previo de TBC fueron factores independientemente asociados a ser PPD positivo al inicio de la carrera. Por otro lado, se encontró que el ingreso reciente a la carrera (periodo 2009-2010 comparado con el periodo 2007-2008) fue un factor de riesgo para conversión de PPD durante los años de seguimiento después de ajustar el modelo por el sexo de los participantes.

Respecto a la prevalencia de PPD positivo al inicio de la carrera, los hallazgos de este estudio muestran resultados intermedios comparados con otros estudios. Por ejemplo, varios estudios han reportado prevalencias menores en estudiantes de medicina: uno efectuado en Irán encontró una prevalencia de $6 \%{ }^{12}$, mientras que otro reportó una prevalencia de 7,7\% en Suiza ${ }^{25}$. Asimismo, un estudio en Río de Janeiro, Brasil, halló 6,9\% de prevalencia, el mismo valor que una investigación en República Checa en el año $2005^{26}$. Por otro lado, otros trabajos han reportado tasas mayores de infección tuberculosa latente: un reporte de Turquía halló una prevalencia de 51,6\% ${ }^{27}$, mientras que un trabajo paralelo realizado en el Hospital Dos de Mayo de Lima (2006) encontró una prevalencia de $20,9 \%$ entre estudiantes de ciencias de la salud ${ }^{28}$. Una de las posibles explicaciones podría estar dada por la diferencia en las incidencias de $\mathrm{TBC}$ en la comunidad entre los diversos países mencionados. El Perú es uno de los países que tiene una de las tasas de morbilidad más alta en el mundo y, por ende, la exposición previa al bacilo tuberculoso es alta, incluso antes de iniciar la carrera médica ${ }^{1,3}$. En general, en el Perú la prevalencia de PPD positivo en población general varía entre 35 y 50\% según estudios previos ${ }^{29,30}$.

Dos factores estuvieron independientemente asociados a una mayor prevalencia de PPD positivo al inicio de la carrera: antecedente de TBC antigua y el año reciente de ingreso a la Escuela de Medicina. Haber tenido TBC antes genera respuesta inmune celular contra el bacilo, lo cual a su vez genera una alta posibilidad de respuesta positiva por hipersensibilidad retardada, clásicamente descrito y usado para el diagnóstico rápido de $\mathrm{TBC}^{31}$. Actualmente, se le atribuye un valor predictor positivo de más de $90 \%$ a la prueba de tuberculina para detectar infección latente o exposición previa ${ }^{32,33}$. Muchos factores podrían explicar el por qué el año de ingreso a la carrera es un factor asociado a positividad a la prueba de tuberculina; entre éstos se encuentran: diferencias en las características de la población de estudio entre aquellas que ingresaron antes y después, edad, nivel socio-económico, historia de contacto TBC, presencia de cicatriz BCG (bacilo de Calmette-Guerin), entre otros ${ }^{28}$. Posteriores estudios son necesarios para evaluar la magnitud e importancia de dichos factores.

La mayoría de estudios previos sobre conversión de PPD han sido realizados con poblaciones de médicos o enfermeras en ejercicio, pero pocos han evaluado el riesgo de los estudiantes de pregrado en los diferentes programas de formación profesional, especialmente durante los primeros años ${ }^{12}$. Los estudiantes de pregrado tienen una exposición gradual al bacilo tuberculoso desde los primeros años de estudio hasta el término de su carrera, es decir, a lo largo de toda su formación profesional. Este estudio encontró una incidencia de conversión en la prueba de tuberculina en estudiantes de medicina cercana a 3\%. Algunos autores han publicado resultados similares. Un estudio realizado en Suiza encontró una tasa de conversión anual de $3,4 \%{ }^{25}$, mientras que otro en Brasil halló una incidencia de conversión anual de $3,9 \% \%^{34}$. En otro estudio efectuado en Perú, se encontró una tasa de incidencia inferior, alrededor de $1,1 \%{ }^{28}$. Otros han encontrado tasas mayores, como un estudio brasileño que reportó una tasa de conversión de 10,5\% anual en estudiantes de medicina ${ }^{35}$. Las diferencias podrían deberse a las discrepancias en el tiempo de exposición hospitalaria; aunque se debe tener en cuenta otras variables influyentes como la situación endémica de TBC en cada país (por ejemplo, zonas de alta incidencia).

La exposición puede variar a lo largo de la carrera médica donde, progresivamente, la exposición a centros hospitalarios aumenta a medida que el estudiante avanza en sus años de formación. En ese sentido, un estudio guatemalteco reportó tasas de conversión más altas, alrededor de $35 \%$, en estudiantes de medicina del quinto al séptimo año de medicina ${ }^{36}$, mientras que una investigación colombiana reportó $16 \%$ en sus estudiantes en práctica clínica $^{14}$. Aunque todos los estudios enrolaron estudiantes de medicina, los datos analizados en el presente estudio son de los primeros tres años de la carrera, en comparación con los estudios previos que involucraron estudiantes con 6 a 8 años de estudios ${ }^{28,34}$. Más aún, se sabe que la exposición hospitalaria persiste aún después de haber culminado los estudios universitarios ya que la exposición se vuelve casi permanente al tener un cargo laboral dentro del centro hospitalario. Por esta razón, estudios previos como el de Bonifacio y cols. muestran tasas de conversión de $17 \%$ en médicos peruanos ${ }^{37}$. Otra publicación reciente (año 2010) proveniente de Argentina, reportó un valor de $10,7 \%$ en trabajadores de salud ${ }^{10}$. La gran diferencia en la incidencia de estos dos estudios es atribuible mayormente a la condición endémica de TBC en esos dos países ${ }^{1,3,5,38}$.

Solamente dos factores estuvieron asociados a la conversión de PPD durante el seguimiento usando modelos bivariados. El año de ingreso a la carrera fue un factor de 
riesgo asociado, especialmente para aquellos que ingresaron en los últimos años, incluso después de ajustar por la variable sexo. Esto implicaría que aquellos alumnos en los primeros años de medicina tienen más riesgo de conversión de PPD que aquellos con más tiempo de estudio, a pesar que la literatura científica llega al consenso de que a mayor tiempo de estudio mayor riesgo de conversión de PPD $^{12,39,40}$. Algunas variables confusoras - como la edad- podrían ser necesarias para modelar adecuadamente nuestros resultados; variables que no pudieron ser colectadas de las historias clínicas. Un IMC $\geq 25 \mathrm{~kg} /$ $\mathrm{m}^{2}$ fue un factor protector para la conversión a la prueba de tuberculina únicamente en el modelo crudo. Algunos estudios han mostrado que las personas con sobrepeso y obesidad tienen un menor riesgo de desarrollo de TBC activa $^{23}$ o de enfermedad grave ${ }^{41}$. Sin embargo, si esta asociación existe, la explicación que la justifica aún no ha sido esclarecida por completo ${ }^{23}$. Estudios más grandes y prospectivos son necesarios para verificar esta asociación, a pesar de que se han descrito asociaciones directas entre TBC y peso ${ }^{12,42-45}$.

Las fortalezas de este estudio radica principalmente en la inclusión de estudiantes de medicina de los primeros años, los que en teoría están menos expuestos al bacilo tuberculoso, así como la presencia de resultados de prevalencia e incidencia y potenciales factores asociados. Sin embargo, este estudio presenta diversas limitaciones. Primero, la ausencia de la edad y de otros factores importantes asociados a la conversión de PPD, como la vacunación con $\mathrm{BCG}$, no pudieron ser evaluados debido al no encontrarse disponibles por la naturaleza retrospectiva del estudio y del análisis de datos secundarios usando registros clínicos. Esto pudo haber limitado la correcta interpretación de los datos. Sin embargo, nuestros resultados en tasas de incidencia están dentro de los valores esperados de acuerdo a la literatura médica. De otro lado, el antecedente de BCG podría estar relacionado a una prueba PPD falsamente positiva, principalmente en los dos primeros años después de la vacunación ${ }^{34,35}$, la cual se da en los primeros años de vida en nuestro país. En países con altas prevalencias de vacunación $\mathrm{BCG}$, un punto de corte de $10 \mathrm{~mm}$ o más para PPD debería excluir cualquier reacción debida a BCG y fue usada en la evaluación de esta cohorte ${ }^{46,47}$. Como consecuencia, creemos poco probable que cualquier conversión pueda deberse a la vacunación $\mathrm{BCG}$ en vez de infección reciente. Segundo, la lectura de PPD, aunque rutinaria, no fue un proceso estandarizado. Finalmente, a pesar de contar con datos de 707 estudiantes, no todos tenían los resultados de PPD por la revisita que hay que realizar para la lectura. No obstante, nuestro estudio muestra resultados comparables a estudios previos e incluso a una revisión sistemática previamente publicada ${ }^{16}$. Nuevos estudios prospectivos, que incluyan las variables apropiadas y todos los años de formación médica profesional, son necesarios para confirmar estos hallazgos.

En conclusión, mientras la prevalencia de los estudiantes de medicina encontrada muestra un resultado promedio comparado con poblaciones similares, internacionales y nacionales, la incidencia de conversión a la prueba de tuberculina encontrada (3\%) es mayor que otras poblaciones. Debido a que los estudiantes de medicina pertenecen a uno de los grupos con mayor riesgo de contagio, la bioseguridad y el uso de medidas preventivas deben ser prioritarios para evitar la infección por el bacilo tuberculoso.

Agradecimientos. A la Escuela de Medicina de la Universidad Peruana de Ciencias Aplicadas-UPC y sus autoridades por brindarnos las facilidades del caso para la adquisición de las historias clínicas y la publicación de este estudio - especialmente a Denisse Champín Michelena, Claudia Mory Arciniega y Soledad Laos. De igual manera, agradecemos la participación y consentimiento de todos los alumnos de la Escuela de Medicina de la UPC que mostraron interés en participar y apoyar del estudio. Agregamos un reconocimiento especial a la contribución de José Alejandro Palacios Flores.

\section{Resumen}

Introducción: Un tercio de la población mundial presenta una infección tuberculosa latente, con 9,4 millones de casos nuevos reportados en el 2009; los estudiantes de medicina tienen de 2 a 50 veces más probabilidad de adquirir la infección. Objetivos: Establecer la prevalencia de PPD positivo basal al inicio de la carrera médica y determinar la incidencia y los factores asociados a la conversión de PPD en alumnos de medicina. Materiales y Métodos: Análisis secundario de datos de una cohorte histórica (2007-2010) involucrando alumnos de medicina de una universidad privada en Perú. Se evaluó la conversión de PPD. Se aplicó un análisis de regresión binomial para cada variable de interés. Resultados: 707 estudiantes fueron seguidos, de ellos 219 (31\%) fueron hombres. La prevalencia basal de PPD positivo fue $14,4 \%$. Se encontró asociación significativa con el año de ingreso 2007-08 $(\mathrm{p}=0,007)$ y antecedente de tuberculosis anterior $(\mathrm{p}=0,02)$. Con un total de 822 personas-año, la incidencia de conversión fue de 2,92 por 100 personas-año (IC95\%: 1,96-4,36). En el modelo bivariado, el año de ingreso a la carrera y el IMC $\geq 25 \mathrm{~kg} / \mathrm{m}^{2}$ estuvieron asociados con conversión de PPD. Sin embargo, en el modelo multivariado, sólo el año de ingreso mostró asociación estadísticamente significativa $(\mathrm{RR}=2,53$; IC95\%: 1,11-5,76). Conclusiones: Existe una prevalencia basal elevada de infección latente en alumnos de medicina. La tasa de incidencia está dentro de los valores esperados y previamente reportados. Se recomienda prestar más atención a las medidas de bioseguridad y prevención en estudiantes de medicina. 


\section{Referencias bibliográficas}

1.- World Health Organization. Global Tuberculosis Control 2010: Epidemiology Strategy Financing. Genova: World Health Organization; 2010.

2.- Fitzgerald D, Haas D W. Mycobacterium tuberculosis. En: Mandell GL, Douglas RG, Dolin R, editors. Mandell, Douglas \& Bennett's Enfermedades infecciosas: Principios y Práctica. $6^{\mathrm{a}}$ ed. Madrid, España: Elsevier España; 2006. p. 2852-86.

3.- World Health Organization: Highlights. TB Country profile Peru: Surveillance and Epidemiology. Ginebra: World Health Organization; 2010.

4.- Horsburgh C R Jr. Priorities for the treatment of latent tuberculosis infection in the United States. N Engl J Med. May 2004; 350 (20): 2060-7.

5.- Del Castillo H, Mendoza-Ticona A, Saravia J C, Somocurcio J G. Epidemia de tuberculosis multidrogo resistente y extensivamente resistente a drogas (TB MDR/ XDR) en el Perú: situación y propuestas para su control. Rev Peru Med Exp Salud Pública. 2009; 26 (3): 380-6.

6.- Mandell G L, Douglas R G, Bennett J E, Dolin R. Mycobacterium tuberculosis. En: Mandell, Bennett, and Dolin: Principles and Practice of Infectious Diseases. $6^{\mathrm{a}}$ ed. New York: Churchill Livingstone, Elsevier; 2005. p. 2857-8

7.- Cox H S, Sibilia K, Feuerriegel S, Kalon S, Polonsky J, Khamraev A K, et al. Emergence of extensive drug resistance during treatment for multidrug-resistant tuberculosis. N Engl J Med 2008; 359 (22): 2399-400.

8.- Bernabe-Ortiz A. La pobreza contra-ataca: Efectos sobre los resultados del TBC-DOTS en el Perú. Rev Med Hered 2007; 18 (3): 179-80.

9.- Accinelli R, Noda J, Bravo E, Galloso Benites M, López Oropeza L, da Silva Caballero J, et al. Enfermedad tuberculosa entre trabajadores de salud. Acta Med Per 2009; 26 (1): 35-47.

10.- Gonzáles C, Araujo G, Agoglia R, Hernández S, Seguel I, Sáenz C. Tuberculosis en trabajadores de salud. Medic B Aires 2010; 70: 23-30.

11.- Fica A, Cifuentes M, Ajenjo M C, Jemenao I, Zambrano A, Febré N, et al. Tuberculosis in healthcare workers. Rev Chilena Infectol 2008; 25 (4): 243-55.

12.- Emadi-Koochak H, Rasoulinejad M, Seyed Alinaghi S, Parsa M, Salehi F, Jam S, et al. Tuberculin skin test conversion among students during their educational course in medical and pharmacy schools: A multiple cohort study. Tanaffos 2009; 8 (4): 33-6.

13.- Fica A, Ramonda P, Jemenao I, Zambrano A, Febré N, Ajenjo C, et al. Tuberculosis en el personal de salud del Servicio de Salud Metropolitano Sur de Santiago, Chile. Rev
Chilena Infectol 2009; 26 (1): 34-8.

14.- Acosta J C, Parra E, Rivera R. Viraje de la prueba de tuberculina (PDD) en estudiantes de Medicina de la Universidad del Bosque luego de iniciar sus prácticas clínicas. Rev E.C.M. División de Investigaciones 2003; 8 (1): 49-59.

15.- American Thoracic Society. Targeted tuberculin testing treatment of latent tuberculosis infection. Am J Respir Crit Care Med 2000; 161: S221-47.

16.- Joshi R, Reingold A L, Menzies D, Pai M. Tuberculosis among health-care workers in low- and middle-income countries: a systematic review. PLoS Med 2006; 3 (12): 494.

17.- Royo F, González M, Moreno M, Pardo S, Prada A, Arnedo P, et al. Evolución de las resistencias a Mycobacterium tuberculosis en la provincia de Castellón. Arch Bronconeumol. 2000; 36: 551-6.

18.- Saldias F, Méndez I, Ramírez D, Díaz O. El riesgo de infecciones respiratorias en el fumador activo y pasivo. Rev Chil Enferm Respir 2007; 23 (3): 179-87.

19.- Lozano J, Plasencia C, Ramos D, García R, Mahíquez L. Factores de riesgo socioeconómicos de la tuberculosis pulmonar en el municipio de Santiago de Cuba. MEDISAN. 2009; 13 (1): 1-6.

20.- Piñate F M, Rovira Avilán J M. Estado actual de la lucha antituberculosa en Venezuela. Gac Méd Caracas 2007; 115 (4): 325-34.

21.- Hsien-Ho L, Ezzati M, Murray M. Tobacco smoke, indoor air pollution and tuberculosis: A systematic review and meta-analysis. PLoS Med 2007; 4 (1): 173-89.

22.- Lönnroth K, Williams B G, Stadlin S, Jaramillo E, Dye C. Alcohol use as a risk factor for tuberculosis-a systemic review. BMC Public Health 2008; 8: 289.

23.- Leung C C, Lam T H, Chan W M, Yew W W, Ho K S, Leung G, et al. Lower risk of tuberculosis in obesity. Arch Intern Med 2007; 167 (12): 1297-304.

24.- Cegielski J P, McMurray D N. The relationship between malnutrition and tuberculosis: evidence from studies in humans and experimental animals. Int J Tuberc Lung Dis 2004; 8 (3): 286-92.

25.- Turk A, Angst F, Steffen R. Tuberculosis infection notification in Swiss medical students during their clinical electives. Int J Infect Dis 2003; 7 (4): 268-73.

26.- Homolka J, Krejbich F, Simecek P. The risk of TB infection in medical students of Charles University. Chest 2005; 128 (4): 402S.

27.- Yorulmaz F, Cağlar T, Erel C, Ozaydin M. Prevalence and annual risk of tuberculosis infection in Edirne, Turkey. Scand J Infect Dis 2002; 34 (9): 654-6.

28.- Hohmuth B A, Yamanija J C, Dayal A S, Nardell E, Salazar J J, Smith Fawzi M C. Latent tuberculosis infection: risks to health care students at a hospital in Lima, Peru. Int J Tuberc Lung Dis 2006; 10 (10): 1146-51.

29.- Pelly T F, Santillan C F, Gilman R H, Cabrera L Z, García E, Vidal C, et al. Tuberculosis skin testing, anergy and protein malnutrition in Peru. Int $\mathbf{J}$ Tuberc Lung Dis 2005; 9 (9): 977-84.

30.- Saito M, Pan W K, Gilman R H, Bautista C T, Bamrah S, Martín C A, et al. Comparison of altitude effect on Mycobacterium tuberculosis infection between rural and urban communities in Peru. Am J Trop Med Hyg 2006; 75 (1): 49-54.

31.- Mack U, Migliori G B, Sester M, Rieder H L, Ehlers S, Goletti D, et al. LTBI: Latent tuberculosis infection or lasting immune responses to M. tuberculosis? A TBNET consensus statement. Eur Respir J 2009; 33 (5): 956-73.

32.- Cabarcos Ortiz de Barrón A, Lado F L, Pestoni C, Lorenzo V. Utilidad de los tests cutáneos (mantoux y prueba de hipersensibilidad retardada) en la infección por el VIH. An Med Interna 2001; 18 (12): 644-9.

33.- Cohn D L, O'Brien R J, Geiter L J, Gordin F M, Hershfield E, Horsburgh C R, et al. Targeted tuberculin testing and treatment of latent tuberculosis infection. MMWR Morb Mortal Wkly Rep 2000; 49 (6): 1-54.

34.- Silva V M, Cunha A J, Kritski A L. Tuberculin skin test conversion among medical students at a teaching hospital in Rio de Janeiro, Brazil. Infect Control Hosp Epidemiol 2002; 23 (10): 591-4.

35.- Maciel Ethel L N, Meireles W, Silva A P, Fiorotti K, Dietze R. Nosocomial Mycobacterium tuberculosis transmission among healthcare students in a high incidence region, in Vitória, State of Espírito Santo. Rev Soc Bras Med Trop 2007; 40 (4): 397-9.

36.- Arellano A, Fuentes J, Campos R. Respuesta a la prueba de Mantoux en un grupo de estudiantes de medicina, antes y después de su rotación hospitalaria. Rev Med Fac de Med Univ Francisco Marroquín 2008; 1 (7): 12-4.

37.- Bonifacio N, Saito M, Gilman R, Vera Quispe C. high risk for tuberculosis in hospital physicians, Peru. Emerg Infect Dis 2002; 8 (7): 747-8.

38.- Abbate E, Ballester D, Barrera L, Brian M, Echazarreta A, Gaitán C, et al. Consenso Argentino de Tuberculosis. Rev Arg Med Resp 2009; 9: 61-99.

39.- Madrigal A, Vázquez M, Arellano G, Prado T, Guzman C, Gallegos A, et al. Reactividad al PPD en estudiantes de ciencias biomédicas de la Universidad Autónoma de Aguascalientes, México, 2005. Enf Infec Ped 2008; 21 (83): 68-74.

40.- Arbaláez M, Ocampo M, Montoya J, Jaramillo L, Giraldo P, Maldonado A, et al. Evaluación de la respuesta a la tuberculina en 
estudiantes del área de la salud. Pan Am J Public Health 2000; 8 (4): 272-9.

41.- Van Lettow M, Kumwenda J J, Harries A D, Whalen C C, Taha T E, Kumwenda N, et al. Malnutrition and the severity of lung disease in adults with pulmonary tuberculosis in Malawi. Int J Tuberc Lung Dis 2004; 8 (2): 211-7.

42.- Palmer C E, Jablon S, Edwards P Q.

Tuberculosis morbidity of young men in relation to tuberculin sensitivity and body build. Am Rev Tuberc 1957; 76 (4): 517-39.

43.- Edwards L B, Livesay V T, Acquaviva F A, Palmer C E. Height, weight, tuberculous infection, and tuberculous disease. Arch Environ Health 1971; 22 (1): 106-12.

44.- Tverdal A. Body mass index and incidence of tuberculosis. Eur J Respir Dis 1986; 69 (5): $355-62$.

45.- Gupta K B, Gupta R, Atreja A, Verma M, Vishvkarma S. Tuberculosis and nutrition.
Lung India 2009; 26 (1): 9-16.

46.- Teixeira E, Menzies D, Comstock G, Cunha A, Kritski A, et al. Latent tuberculosis infection among undergraduate medical students in Rio de Janeiro State, Brazil. Int J Tuber Lung Dis 2005; 9: 841-7.

47.- Dixon W, Collins M. screening and chemoprophylaxis for tuberculosis infection in college populations. J Am Coll Health 1998; 46: 171-5. 